\title{
Mit Ernsthaftigkeit zum Erfolg!
}

\begin{abstract}
Stakholderdialoge sollen die Kommunikation zwischen Unternehmern und den vom unternehmerischen Handeln Betroffenen verbessern. Durch intensiven Austausch können sie Probleme lösen, Verständnis erzeugen, Verhalten ändern und der Nachhaltigkeit auf die Sprünge helfen. Aber nur wenn Unternehmen zur Änderung bereit sind und Dialogergebnisse konsequent umsetzen, sind Stakeholderdialoge sinnvoll und führen zu befriedigenden Ergebnissen.
\end{abstract}

$\mathrm{M}$ Von Niels Ferdinand it dem Begriff Stakeholderdialog verbinden sich sowohl für Unternehmen als auch für ihre Stakeholder viele Erwartungen. Unternehmen suchen den Dialog zu ihren marktlichen und nicht-marktlichen Interessengruppen, da sie sich hieraus neue Lösungsansätze für Probleme der betrieblichen Leistungserstellung und -verwertung erhoffen. Durch die Beteiligung von Stakeholdern wollen sie ihr Image verbessern und die gesellschaftliche Akzeptanz von Strategien und Handlungsplänen steigern. Zunehmend erkennen Betriebe in den unterschiedlichen Ansichten, Ideen und Kenntnissen ihrer Interessengruppen eine wichtige Ressource, die im Rahmen von Dialogen zu Innovationen im Unternehmen führen kann. Langfristig erwarten sie sich den Aufbau vertrauensvoller Stakeholderbeziehungen - und damit einen wichtigen Wettbewerbsvorteil.

Stakeholder haben ihrerseits ein Interesse daran, Ansprüche direkt gegenüber Unternehmen zu kommunizieren. Sie fordern von Unternehmen die Durchführung von Dialogen, um verstärkt Einfluss auf unternehmerische Entscheidungen nehmen zu können.

\section{Enttäuschte Erwartungen}

Den vielfältigen Erwartungen an das Instrument des Stakeholderdialogs steht die derzeitige Praxis entgegen. Insbesondere Betriebe im Mittelpunkt des öffentlichen Interesses und fortschrittliche Nischenunternehmen wenden es zwar seit vielen Jahren an wie der Ölkonzern Shell und der Kosmetikhersteller The Body Shop. Im deutschsprachigen Raum sind Stakeholderdialoge jedoch erst in wenigen Betrieben etabliert und werden nur selten systematisch eingesetzt.

Kostenintensive und zeitaufwändige Dialogverfahren brachten für die Beteiligten häufig nicht die gewünschten Ergebnisse. Enttäuschte Erwartungen führten zur Ablehnung der Verfahren durch verschiedene Interessengruppen. So wurde das Vorgehen der Firma DaimlerChrysler Aerospace Airbus im Rahmen der Auseinandersetzung um die Erweiterung des Hamburger Werkgeländes kritisiert. Insbesondere wenig einflussreiche Stakeholdergruppen wie Anwohner, lokale Landwirte und Tourismusunternehmen sahen in den eingesetzten Dialogen ihre Interessen nicht ausreichend berücksichtigt (1).

Problematisch ist in diesem Zusammenhang die sehr unterschiedliche Verwendung des Begriffs Stakeholderdialog. Sie reicht vom Telefonat zwischen Unternehmensvertreter mit Umweltverband bis hin zu langwierigen Beteiligungsverfahren. Dieses unterschiedliche Begriffsverständnis führt dazu, dass die Kommunikationspartner im Vorhinein schwer absehen können, ob das Verfahren ihr Vertrauen verdient und sich ihr Engagement lohnt.

Wichtige Merkmale von Stakeholderdialogen lassen sich wie folgt zusammenfassen:

- Der Inhalt der Kommunikation sind Probleme oder Themen, die sowohl für das initiierende Unternehmen als auch für seine Stakeholder von Bedeutung sind.
- Die Kommunikationspartner sind Unternehmensvertreter und alle von dem Thema betroffenen Stakeholder. Ihre Auswahl erfolgt auf Basis eines Issue- oder Stakeholdermonitoring, möglichst unter Beteiligung externer Dialogberater und bereits identifizierter Stakeholdervertreter.

- Das Verfahren ist gerecht, ermöglicht die Einbringung aller Meinungen und ist grundsätzlich dazu geeignet, zu einem Konsens zu gelangen.

- Die Kommunikationsform ist dialogisch, es findet ein Austausch von Argumenten mit einem gemeinsamen Sachbezug statt. Die Kommunikationspartner sind bereit, eigene Sicht- und Handlungsweisen an die begründeten Argumente des Gegenübers anzupassen und falls nötig zu ändern. Damit steht auch der Ausgang des Kommunikationsprozesses im Voraus nicht fest (2).

\section{Verbesserung der Reaktion}

Stakeholderdialoge werden heute in den meisten Fällen professionell begleitet. Die Prozesse konnten in den letzten Jahren erheblich verbessert werden (3). Doch auch ein perfekt moderiertes Dialogverfahren wird im Nachhinein nur dann als erfolgreich angesehen werden, wenn sich die von den Beteiligten erwarteten Ergebnisse einstellen. Teilnehmende Stakeholder verlangen nicht nur die operative Umsetzung der vereinbarten Sachergebnisse. Vielmehr ist es für sie ein entscheidendes Erfolgskriterium, dass Dialogverfahren im Unternehmen einen Prozess organisationalen Lernens auslösen, der sich in Strategien, Zielen und Handlungsweisen niederschlägt. Damit ihre Ansprïche dauerhaft berücksichtigt werden, erwarten sie neben der Anpassung von Routinen und Praktiken eine Änderung der zugrundeliegenden Systeme und Strukturen im Unternehmen.

Die Fähigkeit von Betrieben, Ergebnisse von Stakeholderdialogen in solchen Lernprozessen

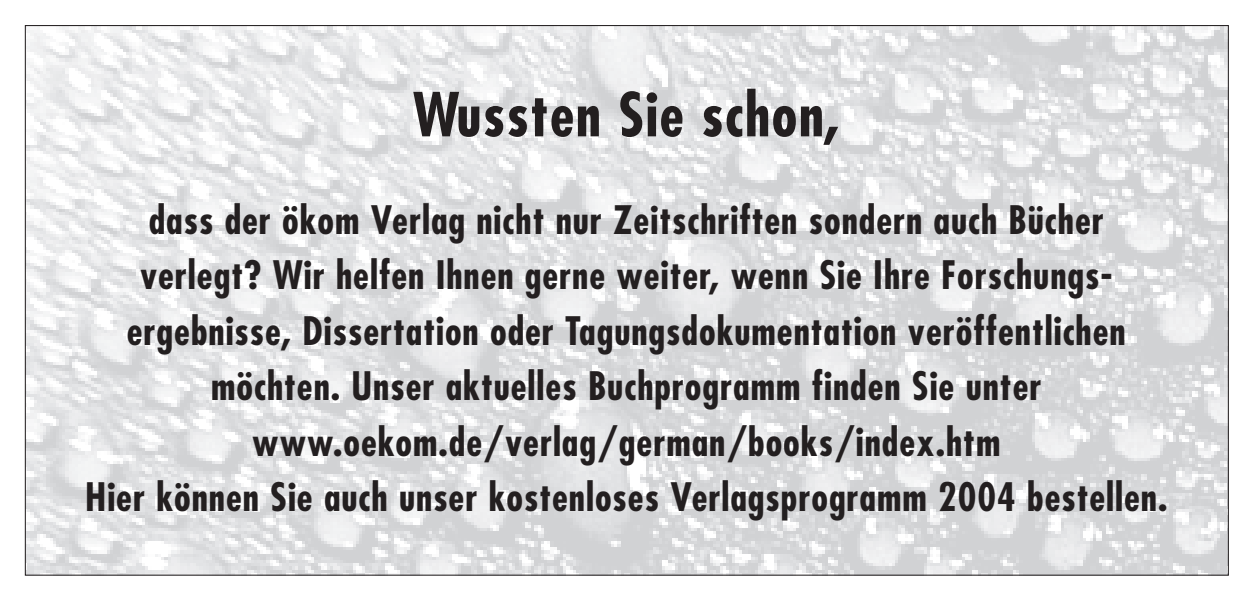


umzusetzen, kann als Responsiveness bezeichnet werden in Anlehnung an die Terminologie des AccountAbility1000 (AA1.000)-Standards. (4).

Die Steigerung der Responsiveness ist eine zentrale Herausforderung für Unternehmen, die das Potenzial von Dialogverfahren ausschöpfen wollen.

Eine umfassende Umsetzung der Dialogergebnisse kann nur gelingen, wenn Unternehmen zu Änderungen bereit sind. Die Integration von Stakeholderansprüchen in Prozesse und Konzepte sollte vor dem Hintergrund der Unternehmensstrategien und -ziele erwünscht sein. Unternehmen ist zu empfehlen, Stakeholderdialoge systematisch einzusetzen und mögliche Probleme einzuplanen. Insbesondere ist der Gefahr der Lähmung von Unternehmensentscheidungen vorzubeugen, denn die Verfahren sind zumeist langwierig und führen häufig nicht zu eindeutigen Ergebnissen (5). Wenn Betriebe nicht an einem Dialog, sondern lediglich am Informationsaustausch mit ihren Stakeholdern interessiert sind, ist dies legitim - solange das Ziel der Kommunikation und der angestrebte Grad der Beteiligung klar kommuniziert werden.

Zur Steigerung der Responsiveness ist es wichtig, Dialogverfahren im Hinblick auf ihre Bedeutung für das normative und strategische Management auszuwerten. Um das Potenzial des Interessenaustauschs zwischen dem Unternehmen und seinen Stakeholdern nutzen zu können, sollten die Resultate bei der Um- beziehungsweise Neuformulierung von Unternehmensleitbildern, -grundsätzen und strategischen Stoßrichtungen berücksichtigt werden. Die Integration der Impulse aus Dialogverfahren kann weiterhin für das strategische Stakeholder- und Kommunikationsmanagement von großer Bedeutung sein.

\section{- Organisationales Lernen fördern}

Die Berücksichtigung nicht-marktlicher Ansprüche in Entscheidungen, der Umgang mit divergierenden Stakeholderinteressen und eine transparente Stakeholderkommunikation sind für viele Unternehmen Neuland. Solche Aspekte müssen erlernt werden, um angemessen auf Stakeholderdialoge reagieren zu können. Eines langwierigen Lernprozesses bedarf es insbesondere zur Ausprägung einer Unternehmenskultur, welche die diversen Ansprüche von Stakeholdern als wertvoll anerkennt. Sie ist entscheidend für die Steigerung der Responsiveness.

Unternehmen, die Dialogverfahren mit verschiedenen Stakeholdergruppen langfristig etablieren und dabei möglichst viele ihrer Mitarbeiter beteiligen, gehen bereits den ersten wichtigen Schritt zu einem gegenseitigen Lernen. Durch die Einbeziehung vielfältiger Sichtweisen, Wahrnehmungen und Erwartungen in Entscheidungen unterstützen sie die Änderung traditioneller Herangehensweisen.

Einen wichtigen Beitrag zur Steigerung der Responsiveness von Unternehmen können die von verschiedenen Autoren vorgeschlagenen Ansätze eines stakeholderorientierten PerformanceMeasurement leisten (6). Solche Systeme machen das Verhältnis von Leistung und Gegenleistung zwischen Unternehmen und ihren Stakeholdern in Form von Kennzahlen mess- und steuerbar. Das hierdurch erreichte Performance-Feedback kann Lernprozesse fördern und die Unternehmensberichterstattung verbessern. Ein stakeholderorientiertes PerformanceMeasurement kanalisiert entscheidungsrelevante Informationen über die Erwartungen von Stakeholdern und unterstuitzt damit ihre Berücksichtigung im operativen, strategischen und normativen Management.

\section{- Ausblick}

Stakeholderdialoge sind nicht der Königsweg für alle Entscheidungssituationen im Unternehmen. Werden sie nicht systematisch vorbereitet, eingesetzt und ausgewertet, können sie Misstrauen und Vorurteile fördern. Fehlt Unternehmen die Bereitschaft zu Veränderungen, besteht die Gefahr, dass sie als „Scheindialoge“ abgelehnt werden. Anderenfalls kann ihr Einsatz jedoch sehr sinnvoll sein - insbesondere zur Lösung komplexer Probleme, die für Stakeholder eine große Bedeutung haben.

In den bestehenden Ansätzen zum stakeholderorientierten Performance-Measurement spielen Dialoge zwischen einzelnen Unternehmen und ihren Interessengruppen zumeist eine untergeordnete Rolle. Damit besteht die Gefahr, dass für die spezifischen Beziehungen ausschlaggebende Faktoren nicht berücksichtigt werden. Es bedarf vor diesem Hintergrund der praktischen Erprobung einer Verknüpfung von Stakeholderdialogen mit dem PerformanceMeasurement.

Für eine zunehmende Zahl von Unternehmen ist heute nicht mehr die Frage ausschlaggebend, ob sie Stakeholder in ihre Entscheidungen einbeziehen sollen, sondern wie eine solche Beteiligung am besten funktioniert. Neben der stakeholderbezogenen Performance wird daher auch die Messung der Wirkung einzelner Methoden des
Stakeholdermanagements immer wichtiger. Für Stakeholderdialoge besteht hier Forschungsbedarf. Die Umsetzung von ihren Sachergebnissen lässt sich relativ leicht im Rahmen eines operativen Controlling nachverfolgen. Da das Potenzial dieses Instruments jedoch gerade in der Änderung von Unternehmensgrundsätzen, Strategien und Zielen liegt, kommt einem strategischen Controlling von Stakeholderdialogen eine große Bedeutung zu. Ansätze hierzu könnten einen wichtigen Beitrag dazu leisten, den Nutzen von Stakeholderdialogen für alle Beteiligten zu steigern und sie dauerhaft zu etablieren.

\section{Anmerkungen}

(1) Vgl. Roloff, J.: Stakeholdermanagement: Ein monologisches oder dialogisches Verfahren? In: Zeitschrift für Wirtschafts- und Unternehmensethik. 3/1, 2002, S. 77-95

(2) Vgl. Rettberg, B.: Der Unternehmensdialog als Instrument einer gesellschaftsorientierten Unternehmensführung: theoretische Fundierung, empirische Untersuchung und Handlungsempfehlungen. Frankfurt a.M. 1999.

(3) Zur Vorbereitung und Durchführung von Dialogprozessen siehe etwa The Environment Council (Hg.): Guidelines for Stakeholder Dialogue. A joint venture. London 1999.

(4) Weitere Informationen: www.accountability.org.uk (5) Vgl. Crane, A./ Livesey, S.: Are you talking to me? Stakeholder communication and the risks and rewards of dialogue. In: Andriof, J./ Waddock, S./ Husted, B./ Sutherland Rahman, S. (Hg.): Unfolding Stakeholder Thinking 2. Relationships, Communication, Reporting and Performance. Sheffield 2003, S. 39-52.

(6) Vgl. etwa Bischof, J.: Die Balanced Scorecard als Instrument einer modernen Controlling-Konzeption. Beurteilung und Gestaltungsempfehlungen auf der Basis des Stakeholder-Ansatzes. Wiesbaden 2002. Zell, M./ Kischewski, J./ Kischewski, S.: Stakeholderorientiertes Informationsmanagement: Weiterentwicklung der Balanced Scorecard zur Stakeholder - Business Process Card. In: Information Management \& Consulting 18 2003/3, S. $66-74$.

\section{Der Autor}

Niels Ferdinand arbeitet als freier Berater und ist externer wissenschaftlicher Mitarbeiter des Center of Research for Management Studies an der Hochschule für Wirtschaft und Verwaltung, Zürich.

Kontakt: Hauptstrasse 216,

CH-7214 Seewis-Schmitten.

E-Mail: niels.ferdinand@unibw-hamburg.de 
(c) 20I0 Authors; licensee IÖW and oekom verlag. This is an article distributed under the terms of the Creative Commons Attribution Non-Commercial No Derivates License (http://creativecommons.org/licenses/by-nc-nd/3.o/), which permits unrestricted use, distribution, and reproduction in any medium, provided the original work is properly cited. 